\title{
Intelligent Healthcare Monitoring in IoT
}

\author{
Kaviya $\mathrm{P}^{1}$, Antony Seba $\mathrm{P}^{2}$ \\ ${ }^{1,2}$ Assistant Professor, Department of Information Technology, Kamaraj College of Engineering and Technology \\ Madurai, Tamil Nadu, India \\ ${ }^{1}$ kaviyaap@gmail.com, 25ebaantony97@gmail.com
}

\begin{abstract}
The developing of IoT-based health care systems must ensure and increase the safety of the patients, their quality of life and other health care activities. We may not be aware of the health condition of the patient during the sleeping hours. To overcome this problem. This paper proposes an intelligent healthcare monitoring system which monitors and maintains the patient health condition at regular intervals. The heart rate sensor and temperature sensor would help us analyze the patients' current health condition. In case of major fluctuations in consecutive intervals a buzzer is run in order to notify the hospital staff and doctors. The monitored details are stored in the cloud "ThingSpeak". The doctor can view the patient health condition using Virtuino simulator. This system would help in reducing the random risks of tracing a patient medical highly. Arduino UNO is used to implement this intelligent healthcare monitoring system.
\end{abstract}

Keywords- Arduino UNO, Heart Rate Sensor, Temperature Sensor, Buzzer, ThinkSpeak, Virtuino simulator.

\section{INTRODUCTION}

Nowadays Internet of Things based health care systems play a significant role in Information and Communication Technologies. It has a remarkable contribution in the development of medical information system. The tracking, tracing, and monitoring of the patients health care activities are challenging research directions. With the development of world, Health monitoring system is used every field such as hospital, home care unit, sports. This health monitoring system use for chronicle diseases patients who have daily check-up. Normally it is difficult to keep track on abnormalities in heartbeat count for patient itself manually. The average heartbeat per minute for 25-year old ranges between 140-170 bpm while for a 60 -year old it is around between 115-140 bpm and body temperature is 37degree Celsius or 98.6 Fahrenheit. Patients are not well versed with manual treatment which doctors normally use for tracking the count of heartbeat. There are various instruments available in market to keep track on internal body changes.

Different platform like Microcontrollers are used to design the system based on this performance. Different biomedical sensors like temperature sensor, heart rate sensor are used for monitoring the health condition which is integrated on single system on chip. If any varied change takes place it is notified.

This notification would help to take an appropriate action at an instance of a time. This would save patients from the future health problem which would arise. This would also help patient's concern doctor to take an appropriate action at proper time.

Another issue, we may not be aware of the health condition of the patient during the sleeping hours. To overcome this problem, this paper proposes an intelligent healthcare monitoring system which monitors and maintains the patient health condition at regular intervals. The heart rate sensor and temperature sensor would help us analyze the patients' current health condition. These two sensors are used to monitor the health condition of the patients especially at the hospital at equal intervals. In case of major fluctuations in consecutive intervals a buzzer is run in order to notify the hospital staff and doctors. These monitored details are stored in the cloud "ThingSpeak". The doctor can monitor the patient health condition through Virtuino simulator using the IP address of the cloud. This project would help in reducing the random risks of tracing a patient medical highly.

The rest of the paper is categorized as follows. We discuss previous works on related topics in Section II. Section III describes proposed system in detail. Section IV provides the system methodology. Section $\mathrm{V}$ provides the results. Section VI finally concludes the paper.

\section{RELATED WORKS}

Ghulam Muhammad, et al [1] have proposed an automatic voice disorder detection system is used to monitor the resident of all age group and professional backgrounds. To analyze the difference between intensity of normal and disorder subjects, the LP co- efficient based spectrum is computed. This leads to high risk of prevalence of voice disorders. Voice complications have the negative impacts over the life of individuals. A smart health care system can assist the residents of different professions and of different age groups. To avoid these circumstances, a smart healthcare system is proposed and developed. The proposed system detects the voice disorder by determining the source signal from the speech through the linear 
prediction analysis. The analysis calculates the features from normal and disordered subjects. The proposed system used the computed spectrum to differentiate between normal and disordered subjects.

Prashant Salunke, et al [2] have proposed a Patient's physiological information is managed and recorded for long time using wearable sensors. This system is expected to reduce costs, increase the quality of life, and enrich the user's experience. This system reduces the time of patient to visit to doctor every time he/she needs to check ECG and temperature and pulse oxygen in blood. Doctors and hospitals could make use of real-time data collected on the cloud platform to provide fast and efficient solution. Doctors who are overwhelmed with patient load can use this system and also it is beneficial for rural patients who have less access to health care facilities.

Sumalatha, et al [3] have proposed an IoT based Health Monitoring System which demonstrates collection and interoperation of data. The data provided to the doctor can support the emergency medical service for a patient for the improvement of healthcare. The sensors and Raspberry Pi 3 Model B are the physical objects equipped for communicating with the doctor though internet. It helps the doctor to control the overall state of monitored patients independently and easily. Also the patient information can be monitored from anywhere in the world. The system comprises of web server part. The sensors gather the medical information of the patient that include patient's heart rate, blood pressure, and pulse rate and the physical examine of the patient through the Pi camera, and this information is sent to the Internet through Raspberry Pi which acts as a server.

Prabha Sundaravadivel, et al [4] have discussed an architecture for an ambulatory health monitoring system using a body coupled communication channel. The proposed architecture can be used for smart health monitoring as part of the Internet of Things. The design was validated using Simulink. A $31 \%$ power reduction was observed in the monitoring system when human body communication happened.

S.Amutha et al [5] have proposed an IoT based wireless healthcare monitoring. They have developed a system for remote patient monitoring in healthcare field. In hospitals, patient's physiological parameters are needed to be constantly monitored, usually done by a doctor or other paramedical staff for maintaining a record of it. It is a tedious method. The primary function of this system is to constantly monitor inpatients and outpatients physiological parameters such as pulse rate, temperature using wireless communication. This system continuously reads patient's pulse rate or heart beat rate and body temperature through Fingertip Measuring Heart rate sensor and Thermistor Temperature Sensor. This system enables high risk patients to be timely checked and enhance the quality of a life of patients.

\section{PROPOSED WORK}

In this work, we monitor the heart beat and body temperature using the heart rate sensor and temperature sensor (LM35). The data are collected and stored in the cloud "ThingSpeak". In case of abnormal change in the heart rate or body temperature the buzzer in the system alerts the nearby person and sends a message to the consultant doctor's mobile phone. The stored data in cloud can be viewed by using mobile application. Virtuino simulator which shows the current patient's condition to the doctor from anywhere.

The system is a combination of hardware and software components. The hardware part consists of embedded system and software is the mobile app designed using Android Studio.

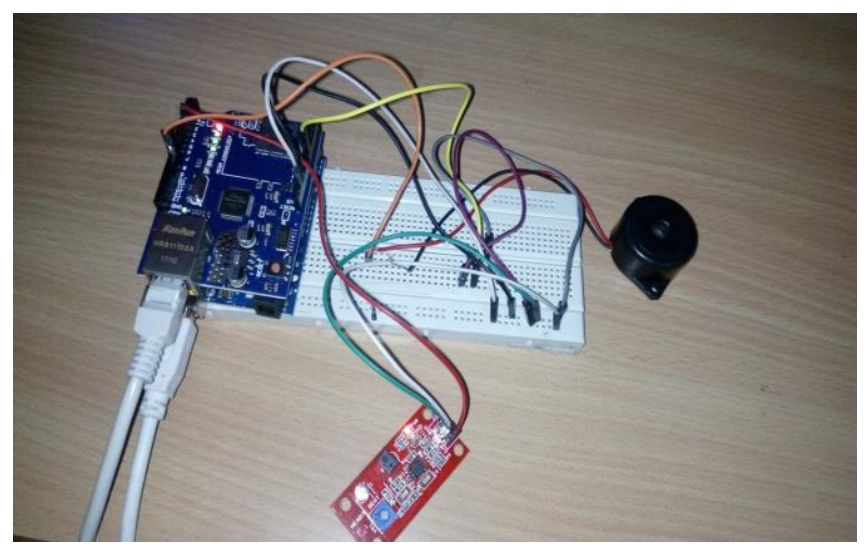

Fig. 1: Hardware Connection

\subsection{Reading Inputs}

\subsubsection{Temperature Sensor}

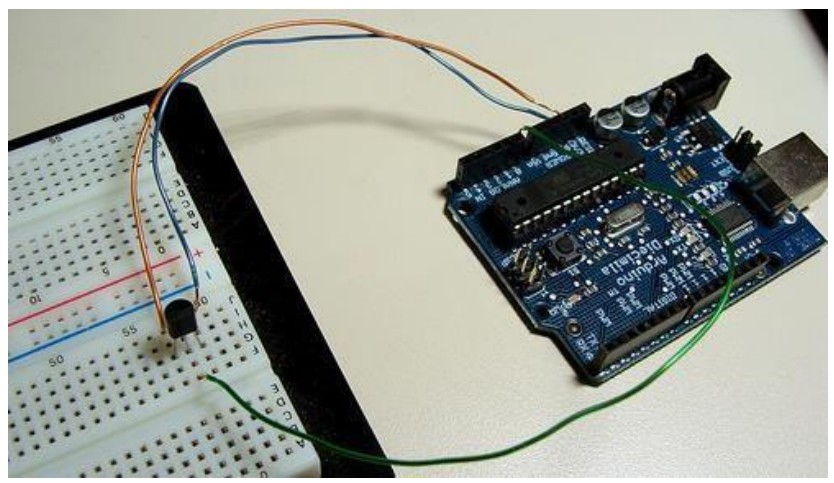

Fig. 2: Temperature Sensor Connection

Fig.2 shows the connection between the Arduino and LM35. Here the green colour wire is connected to the analog pin A1, orange colour wire is connected to Vcc and blue colour wire is connected to ground in the arduino board. 
ALGORITHM

1. Collect body temperature as degree Celsius or degree of Fahrenheit.

2. Convert voltage $->$ Celsius

3. Temp $=($ output voltage $/ 1024) * 5000$

4. Cel=Temp / 10

5. Finally display the output in the form of ' $\mathrm{C}$.

\subsubsection{Heart Rate Sensor}

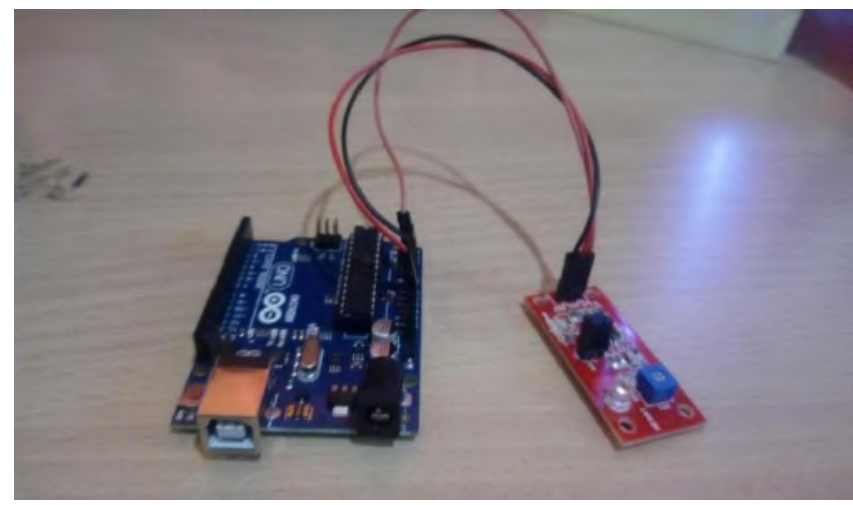

Fig. 3: Heartbeat Sensor Connection

Fig. 3 shows the connection between the Arduino and heart beat sensor. Here the pink colour wire is connected to the analog pin A1, Red colour wire is connected to Vcc and black colour wire is connected to ground in the arduino board.

\section{ALGORITHM}

1. Collect the heart rate from the patient.

2. Check the conditions

$$
\begin{gathered}
\text { if }((\text { millis }() \text { - oldTime })>1000) \\
\text { beat }=\text { beatCount } * 60 ; \\
\text { oldTime }=\text { millis }() ; \\
\text { beatCount }=0 ;
\end{gathered}
$$

3. Increment the count value

4. Finally the value is displayed in the serial monitor.

\subsection{Processing the Data}

It analyze the inputs and checks the condition whether the heartbeat, body temperature are normal or abnormal. To display the temperature results on the web server, a ThingSpeak is used.

\subsection{Emergency Alerts and Mobile App}

If there is a change in the body temperature and heartbeat, the buzzer alters the nearby person and the message will send to the doctor. Doctor can continuously monitor the patient's health condition on his smart phone using a Mobile app.

The patients' history will be stored in the cloud platform. The doctor can access the information from anywhere at any time. Using the IP address of the ThingSpeak, the doctor can view the current health status of the respective patient.

\section{SYSTEM ARCHITECTURE}

Temperature sensor and heart rate sensor are used to sense the patients' health condition. Sensors are connected to the Arduino microcontroller which controls the signals. If there is a change in the body temperature and heart beat, the buzzer alerts the nearby people. As soon as the buzzer rings, the message will send to the corresponding doctor.

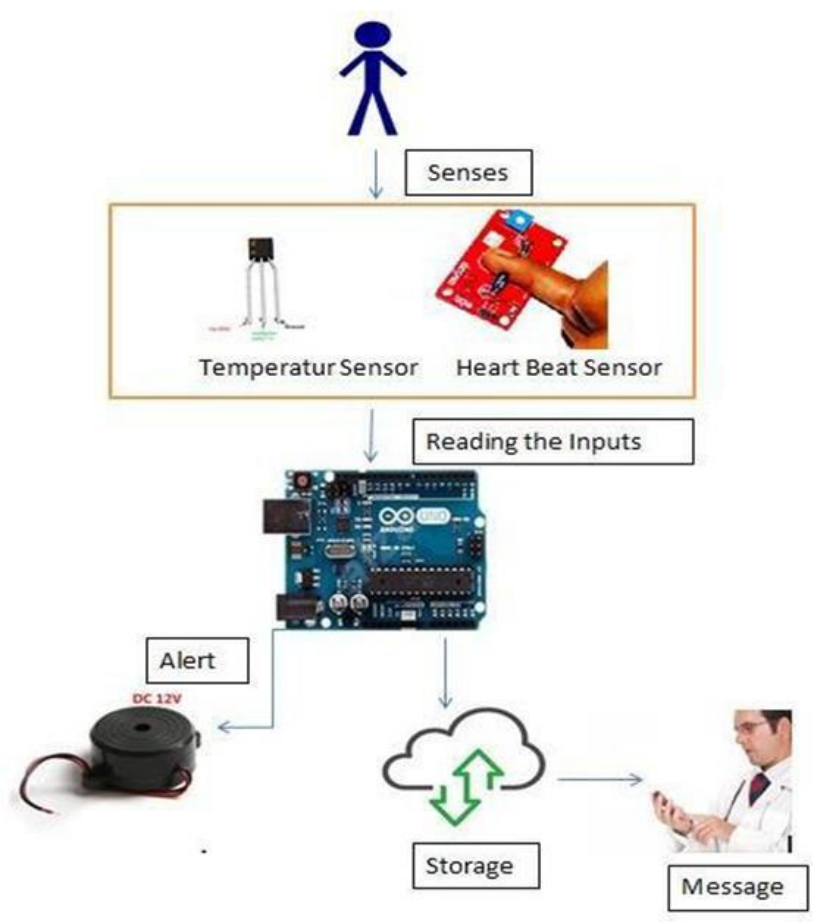

Fig. 4: System Architecture

The sensed data are sent to the cloud (i.e.) ThingSpeak. It is an open source to store and retrieve and the data are been stored in a publically. The data are represented in the form of graph or excel sheet.

The doctor can view the information of the patient at anytime and anywhere using Virtuino Simulator. This Simulator is in-build app in play store. By using the IP address of ThingSpeak channel the doctor can view the current condition of the patient. In this app, it shows the recent temperature and heart beat status of the respective patients.

Finally the doctor can view the patients' details at anytime and anywhere using the Virtuino simulator and ThingSpeak. Health monitoring system architecture is shown in Fig. 4.

\section{RESULT AND DISCUSSION}

\subsection{Temperature Reading}

Fig. 5 shows the temperature result which is displayed on the serial monitor. The resultant data will send to the cloud for doctor's reference. 


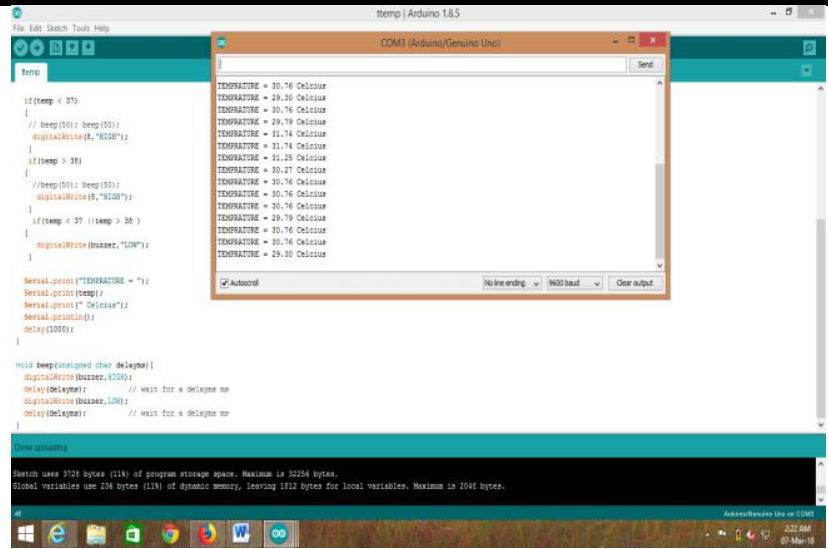

Fig. 5: Temperature Results

Table 1 shows the condition of the human body temperature. If the temperature level increases above $42^{\prime} \mathrm{C}$ or decreases below $25^{\prime} \mathrm{C}$, buzzer alerts the nearby person and message send to the doctor's concern mobile phone.

Table. 1: Temperature data

\begin{tabular}{|c|c|}
\hline Temperature & Causes \\
\hline$<25{ }^{\prime} \mathrm{C}$ & Low \\
\hline$>42^{\prime} \mathrm{C}$ & High \\
\hline $37^{\prime} \mathrm{C}$ & Normal \\
\hline
\end{tabular}

\subsection{Heart Beat Reading}

Table 2 shows the condition of the patients' heart beat. If the heart beat level increases above 120 BPM or decreases below 60 BPM, buzzer alerts the nearby person and message send to the doctor's concern mobile phone.

Table. 2: Heart Beat Data

\begin{tabular}{|c|c|}
\hline Heart Beat & Causes \\
\hline$<60 \mathrm{bpm}$ & Low \\
\hline$>120 \mathrm{bpm}$ & High \\
\hline $60-120 \mathrm{bpm}$ & Normal \\
\hline
\end{tabular}

Fig. 6 gives the heart beat result which is displayed on the serial monitor. The resultant data are sent to cloud for doctor's reference.

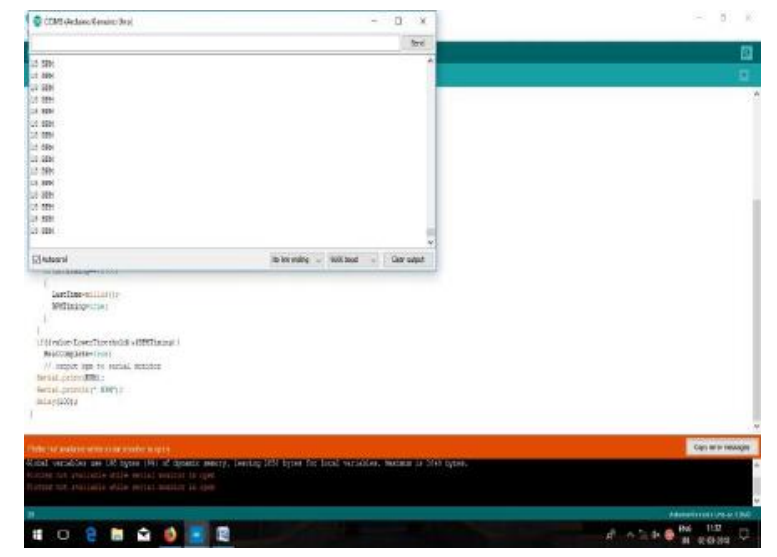

Fig. 6: Heart Beat Results
Table 7.2 shows the condition of the patients' heart beat. If the heart beat level increases above 120 BPM or decreases below 60 BPM, buzzer alerts the nearby person and message send to the doctor's concern mobile phone.

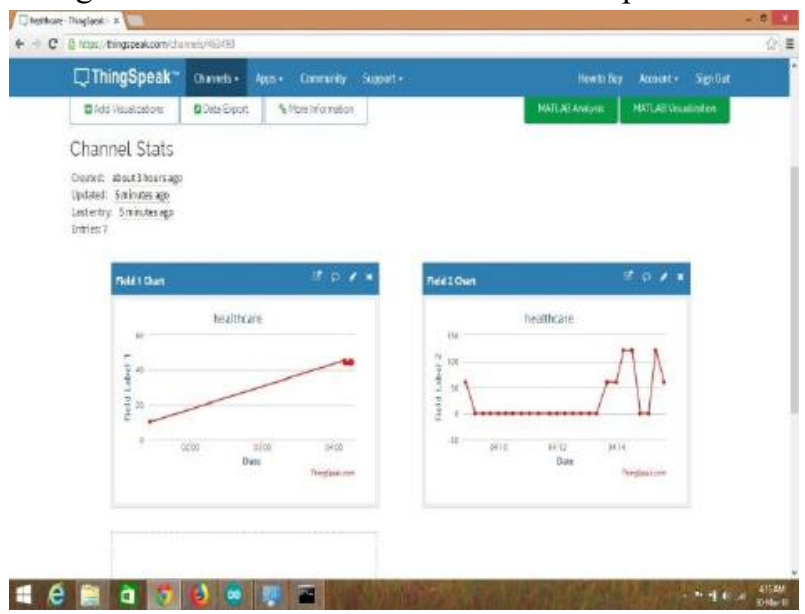

Fig. 7: Data in ThinkSpeak

The data are collected from the system and pushed into the ThingSpeak. In ThingSpeak, we created two channels. Temperature values are stored in first channel whereas heart beat values are stored in second channel.

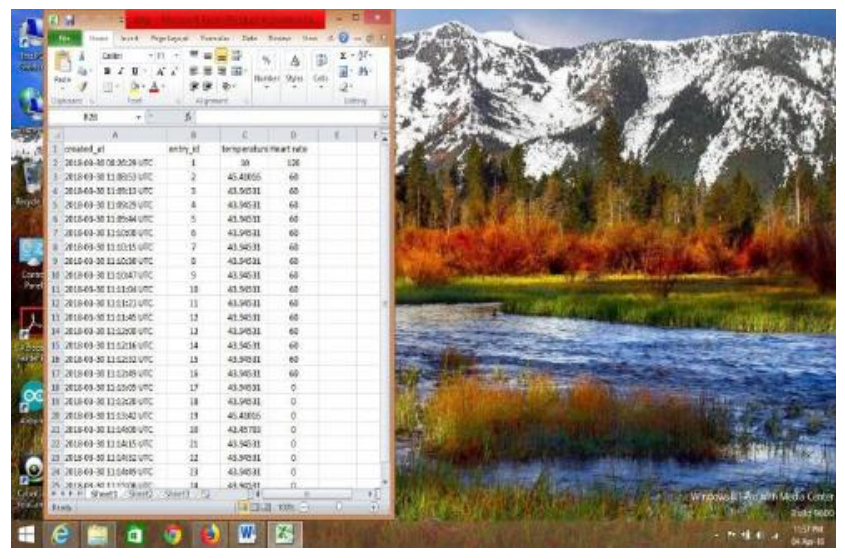

Fig. 8: Data in ThinkSpeak (.CSV)

In Fig. 8, the data are exported in .csv file format to view the health condition of the patients' as show in the Fig. 7.
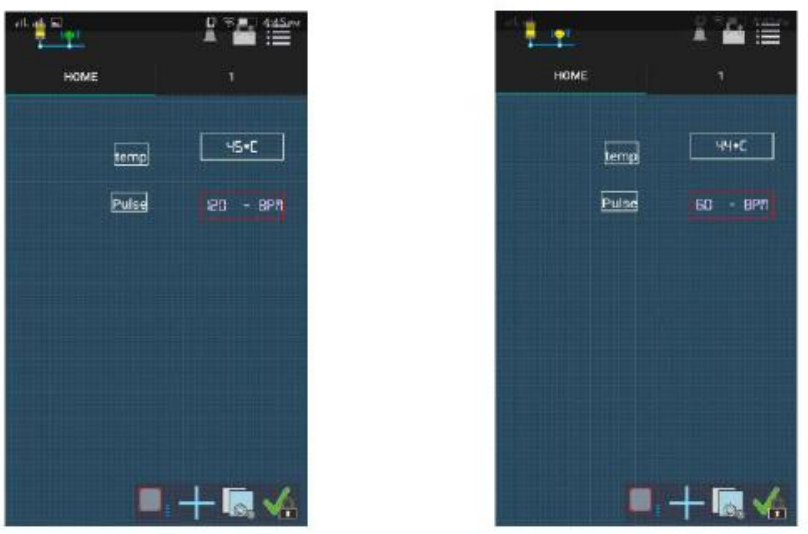

Fig. 9: Virtuino Simulator Results in Mobile 
Virtuino is an Android app for monitoring sensors or control electrical devices via Bluetooth, local Wi-Fi or Internet. In this Simulator, doctor can check the current heart beat and temperature value for the patient. Figure 7.6 shows the current value of the patients' health conditions. It can be viewed through the doctor's mobile phone by using the IP address.

\section{CONCLUSION}

The proposed health monitoring system monitors and tracks the patients' health conditions like body temperature and heartbeat periodically. The buzzer in the system alerts the nearby person and the message is send to the consultant doctor's mobile phone in case of any emergency. The data are stored in the cloud "ThingSpeak". Hence, the health tracking professionals can monitor the patients from anywhere at any time. Thus our intelligent health monitoring system is able to transmit the sensed data to the doctor's PC or mobile Phone. This project would help in reducing the random risks of tracing a patient medical highly.

\section{REFERENCES}

[1] Antonio J. Jara, Miguel A. Zamora-Izquierdo, and Antonio F. Skarmeta. (2015). Interconnection Framework for Health and Remote Monitoring Based on the Internet of Things, IEEE Journal On Selected Areas In Communications/Supplement, Vol. 31, No. 9.

[2] Chi-chun Lo, Tsung-yiChien, Jeng-shyang. (2016). Novel Non-Contact Control System for Medical Healthcare of Disabled Patients, Received April 1, 2016, accepted April 13, 2016, date of publication May 11, 2016, date of current version October 6,.

[3] IulianaChiuchisan, HaritonNicolaeCostin,OanaGeman. (2014). Adopting the Internet of Things Technologies in Health Care Systems, 2014 International Conference and Exposition on Electrical and Power Engineering (EPE 2014).

[4] Natarajan.K. (2016). Smart Health Care System Using Internet of Things, Journal of Network Communications and Emerging Technologies (JNCET) Volume 6, Issue 3, ISSN: 2395-5317.

[5] PrabhaSundaravadivel and Saraju P. Mohanty. (2016). Exploring Human Body Communications for IoT Enabled Ambulatory Health Monitoring Systems, IEEE International Symposium on Nanoelectronic and Information Systems (iNIS), DOI: $10.1109 /$ iNIS.2016.016.

[6] Prashant Salunke and RasikaNerkar. (2017). IoT Driven Healthcare System for Remote Monitoring of
Patients, International Journal for Modern Trends in Science and Technology Vol. 03, Issue. 06.

[7] Saraswathi, S. Amutha. (2016). "IoT Based Wireless Healthcare Monitoring, IJETST-Vol.03., Issue 05May ISSN 2348-9480.

[8] Sumalatha V and Dr. R. C. Biradar. (2016). IoT based Health Monitoring System, IJRT Vol.05., Issue. 05.

[9] Zainab H. Ali. (2017). Internet of Things (IoT): Definitions, Challenges and Recent Research Directions, International Journal of Computer Application, Vol.128., Issue.01., DOI: 10.5120/ijca2015906430.

[10]Zulfiqar Ali, Ghulam Muhammad, And Mohammed F. Alhamid. (2016). An Automatic Health Monitoring System for Patients Suffering From Voice Complications in Smart Cities, IEEE International Conference on Multimedia \& Expo Workshops (ICMEW), DOI: 10.1109/ICMEW.2016.7574719. 